\title{
Metodología PACIE en la Educación Virtual: una experiencia en la Universidad Técnica del Norte
}

\author{
Andrea V. Basantes, Miguel E. Naranjo y Vivian. Ojeda \\ Universidad Técnica del Norte, Campus. Avenida 17 de Julio, 5-21, General José María Córdova, Código \\ postal 100105, Ibarra, Ecuador. (e-mail: avbasantes@utn.edu.ec, menaranjo@utn.edu.ec, \\ vojeda@utn.edu.ec,)
}

Recibido Jun. 15, 2017; Aceptado Ago. 11, 2017; Versión final Oct. 10, 2017, Publicado Abr. 2018

\begin{abstract}
Resumen
Esta investigación tuvo como objetivo identificar las potencialidades de la metodología PACIE (Presencia, Alcance, Capacitación, Interacción, E-learning) aplicada en el entorno virtual de aprendizaje de la Universidad Técnica del Norte en Ecuador. Es una investigación desde un enfoque mixto (cualicuantitativo), se utiliza el método científico y corresponde a un estudio descriptivo de corte transversal. La recolección de la información se realizó a través de encuestas online, de entrevistas a profundidad a expertos, de análisis documental y de la triangulación de la información. Los resultados arrojan que para matricularse en un curso virtual inciden los criterios metodología, capacitación docente, documentación de apoyo, duración y prestigio institucional. En conclusión, las potencialidades de PACIE fortalecen el programa curricular online, el trabajo colaborativo y promueve la calidad y calidez humana, características percibidas y valoradas por los estudiantes.
\end{abstract}

\section{PACIE Methodology in Virtual Education: an experience at Técnica del Norte University}

\begin{abstract}
This work aimed to identify the potential of PACIE methodology (Presence, Achieving, Capacity development, Interaction, E-learning) applied in the virtual learning environment at the University Técnica del Norte in Ecuador. This is a research with a mixed (qualitative-quantitative) approach that uses the scientific method and is of the type called cross-sectional study. Data collection was done using online surveys, in-depth interviews to experts, documentary analysis and data triangulation. The results show that to get enrolled in a virtual class, aspects such as methodology, teacher training, support documentation, duration, and institutional prestige have influence. In conclusion, PACIE's potential strengthens online curriculum, collaborative work and fully promotes human quality and warmth, characteristics that are perceived and valued by students.
\end{abstract}

Keywords: PACIE; ICT; virtual learning; virtual environment 


\section{INTRODUCCIÓN}

La expansión de la llamada Sociedad del Conocimiento en la vida moderna, se ve reflejado en el intenso intercambio de información a escala mundial de forma competitiva, dinámica, variable y compleja (Castro y González-Palta, 2016), lo cual demanda la implementación de fórmulas organizacionales y tecnológicas en las universidades para lograr una formación de calidad no sólo en la profesionalización de una carrera universitaria, sino también a la formación humana y creativa que permita enfrentar con solvencia los retos de la vida (Rocha, 2013). Los cambios determinados en gran medida por el desarrollo de las Tecnologías de Información y Comunicación hacen que la sociedad del conocimiento precisa un saber, uso y dominio adecuado para gestionar debidamente las habilidades propias de la era digital (Cózar-Gutiérrez et al., 2016). Sin embargo, la relación entre la evolución tecnológica y la evolución social, exigen un desafío a las instituciones de educación superior en su papel fundamental de formación que comprende la generación, conservación, intercambio y transferencia de conocimientos.

El fortalecimiento de la Educación Superior es una exigencia en estos tiempos de gran avance tecnológico, constituye prioridad la incorporación de estrategias tecno pedagógicas que permitan gestionar, administrar, distribuir, monitorear, evaluar y apoyar las diferentes actividades de formación; por consiguiente, los Entornos Virtuales de Aprendizaje (EVA) se han convertido en una herramienta de interacción cotidiana en la vida universitaria y va logrando un papel primordial en la práctica de enseñanza-aprendizaje. La preocupación por vincular las nuevas formas educativas con las tecnologías digitales ha sido abordada por varios autores (Micheli y Armendáriz, 2011; Mejía y López, 2016; García, 2017; Sánchez, 2017), la educación virtual elimina las fronteras "espacio y tiempo" de las formas tradicionales de enseñanza. En correspondencia con esta tendencia, las universidades latinoamericanas están inmersas en el desarrollo e implementación de estrategias para potenciar sus entornos virtuales. Según el informe sobre la perspectiva económica de América Latina del año 2017, el acceso a la educación superior se ha ampliado, pero aún es insuficiente en la región; de los jóvenes emprendedores latinoamericanos solo el 13\% tiene educación universitaria; por consiguiente, la educación superior permanece sin explotar (OCDE, Naciones Unidas y CAF, 2016). Como dinámica de su proyección a la sociedad nace la propuesta de educación virtual como una estrategia para aprovechar sus potencialidades a través de las plataformas tecnológicas existentes.

La Reunión Internacional de las Cátedras UNESCO del Sector Educación del 2014, en su reflexión sobre los desafíos que confrontan los sistemas de educación superior, abordó el tema de los programas virtuales de enseñanza y el apoyo que este organismo brinda a América Latina para el impulso de este tipo de enseñanza en la región (UNESCO, 2014). Además, la UNESCO está tratando los temas de "Universidad para el Desarrollo", y sugiere que las universidades deben impulsar procesos interactivos de aprendizaje para la innovación, a los que contribuirán desde los conocimientos y las capacidades en el desarrollo integral del ser humano a partir de las necesidades que surjan para que las innovaciones resulten tecnológica y socialmente satisfactorias (Arocena y Sutz, 2016). Para avanzar en la senda de la sociedad del conocimiento, los países de América Latina y el Caribe deben masificar aún más sus sistemas de educación terciaria, robustecer su capacidad científica y tecnológica, y conectarse mejor con su sociedad. El primer desafío de los sistemas de educación terciaria de la región es asegurar la equidad de su expansión a través de la promoción y movilidad social. El segundo desafío es responder a las nuevas exigencias que la globalización, la sociedad de la información y la economía del conocimiento en la cultura digital imponen a los países en vías de desarrollo a fin de generar una capacidad propia de producción científica y tecnológica. Un último desafío que enfrentan los sistemas de educación superior Latinoamericanos es conectarse mejor y abrirse a sus propias sociedades (OREALC, 2014).

En los países desarrollados los conocimientos derivados de la investigación y la docencia en los niveles más altos de la educación superior influyen cada vez en mayor medida, en amplios segmentos de la producción y los servicios. Se trata de generar con mayor velocidad conocimientos que puedan competir con los demás; este es el caso de las universidades europeas, estadounidenses, canadienses y asiáticas, se interesan por producir conocimientos científicos a partir de la investigación y la docencia; de manera que, estos tengan impacto en la competitiva senda del mejoramiento de productos y servicios. En el caso de Latinoamérica, se acercan a esta estrategia las universidades de Brasil, Argentina, Chile y México (Marsiske, 2015); pero todavía distan mucho el resto de las universidades de la región.

Debido a la limitada oferta académica en el entorno virtual latinoamericano, las universidades "proveedoras" que cuentan con infraestructura, tecnología y respaldo político como es el caso de Asia, Europa y Norteamérica, ven a Latinoamérica como un mercado potencial. Por consiguiente, la educación virtual en nuestra región necesita fortalecerse para enfrentar desafíos del mundo globalizado y formar profesionales pertinentes que den respuestas a las problemáticas de la región. Fortalecerse en esa dirección supone un desafío a la universidad latinoamericana, que debe tener una propuesta de educación virtual que muestre pertinencia, relevancia, actualidad y debe cumplir con los cánones de dinamismo, información veraz, conocimiento actualizado y práctico, y otras tantas exigencias de este tipo de enseñanza. 
Con este contexto, Cano (2012) expresa que, América Latina va cobrando fuerza en la educación masiva a través de las TIC. Ejemplo de ello es el proyecto @Lis-Integra, donde convergen y forman parte del mismo, países como España, Irlanda, Italia, Uruguay, Argentina, y Chile, con más de 20 proyectos encaminados a la utilización de entornos virtuales de aprendizaje mediados por las nuevas tecnologías de información y comunicación en actividades educativas y administrativas. Cabe señalar que universidades de varios países como Chile, Argentina, México y Perú, ya ofertan cursos de formación online.

En este sentido, la universidad virtual asume el uso de las TIC en los nuevos procesos de aprendizajes y adecúa la enseñanza a los nuevos escenarios en los que conviven gran parte de los estudiantes hoy en día. Martínez y Fernández (2011) mencionan que, existen múltiples ventajas del uso del entorno virtual en la enseñanza; entre ellas: la manipulación y exploración de fases de aprendizaje; la reflexión del proceso de aprendizaje llevado a cabo; la acomodación de los conocimientos nuevos a los ya adquiridos; la colaboración, discusión y cooperación con el resto de los estudiantes del aula y la extrapolación consciente de los conocimientos aprendidos a un contexto futuro de trabajo.

La expansión de internet y las TIC han transformado radicalmente la forma de aprender e interrelacionarnos en los diferentes ámbitos del contexto contemporáneo; ya que estas proveen un sin número de herramientas, recursos, medios y formatos que posibilitan estrategias didácticas para facilitar la construcción de conocimientos (Basantes et al., 2017); sin embargo, la posibilidad de utilizar la tecnología emergente en el proceso de enseñanza-aprendizaje provocó que los entornos virtuales de aprendizaje replicaran el paradigma conductista, con niveles de sobreabundancia de información, estableciendo propuestas de formación virtual sin ningún tipo de rigurosidad. En este sentido, se priorizó a la tecnología sobre la pedagogía, la comunicación se relegó a segundo plano y la tasa de deserción de los estudiantes fue sumamente alta. Jordan (2015) menciona que, solo un $15 \%$ de los estudiantes concluyen los cursos online (Yamba-Yugsi, y Luján-Mora, 2017), en consecuencia, se debilitó el nivel de aceptación de la educación virtual.

En otro orden está el fenómeno de diversidad de actitudes ante el uso de las tecnologías. La mayoría de los estudiantes están comprendidos en edades que los identifican como nativos digitales, es decir que tienen una gran familiarización con el mundo virtual, forma parte de su modo de vida. Sin embargo, en el caso de los docentes, en concordancia con Flores (2017), son migrantes digitales y es mayor el esfuerzo que se debe emplear para apropiarse de estas nuevas formas de comunicación, y más allá de esa apropiación para lograr el uso de las mismas como herramienta de enseñanza-aprendizaje. En este sentido las universidades deben ser cuidadosas con la propuesta de enseñanza en el entorno virtual evidenciando la veracidad del conocimiento a través de una metodología adecuada que contemple la experticia pedagogía y técnicas didácticas que brinden garantía a los procesos de enseñanza y aprendizaje. Como parte de la solución a esta problemática el autor ecuatoriano Pedro Camacho crea la metodología PACIE (Oñate, 2009), con un enfoque trascen-dental en el acompañamiento y seguimiento al estudiante basado en la calidad y calidez humana; así como también impulsa la posibilidad de innovar las estrategias didácticas, el trabajo en equipo, el aprendizaje constructivo y colaborativo para intercambiar información y coadyuvar un aprendizaje significativo.

La metodología PACIE aplicada en un entorno virtual de aprendizaje contribuye a alcanzar los objetivos del proceso de enseñanza aprendizaje mediante la incorporación de la TIC de forma gradual y reflexiva, aspectos considerados claves en la formación online, ya que requiere de compromiso, responsabilidad, voluntad y una fuerte disposición para el autoaprendizaje; por consiguiente, la demanda de un ser humano con una formación integral y contextualizada, a la altura de los niveles de desarrollo de la diversidad contemporánea, reclama una revolución continua en la formación de profesores universitarios (Acosta et al., 2015); es decir, el docente debe estar capacitado para proporcionar las herramientas idóneas y permitan lograr los objetivos planteados. Según Martínez y Fuentes (2014), la metodología PACIE es un modelo que modifica el rol docente por una acción tutorial eficiente, quién desde un sentido humanizante motiva y realiza el acompañamiento necesario para disminuir los índices de deserción en los cursos de enseñanza virtual, que cómo se mencionó anteriormente constituye una de las falencias del sistema educativo online. En la Universidad Técnica del Norte, existe la experiencia de la aplicación desde un principio, de la metodología PACIE en el entorno de aprendizaje virtual. Esto ha permitido incorporar las TIC con un enfoque tecnopedagógico basado en una metodología que nace en el entorno latinoamericano tomando en cuenta las dimensiones socioculturales de los actores involucrado en el proceso de enseñanza aprendizaje.

En síntesis, PACIE busca sumar las tecnologías y situar al docente como impulsor de los procesos de aprendizaje (Núñez, 2015), orientando el proceso desde un enfoque constructivista de temas y contenidos teóricos, pero también, velando la estructura grupal y las relaciones que allí se establecen como un componente esencial en la formación humana (Lerner y Gil, 2006). En correspondencia, esta investigación tuvo como objetivo identificar las potencialidades de la metodología PACIE aplicada en el entorno virtual de aprendizaje de la Universidad Técnica del Norte en Ecuador. 


\section{METODOLOGÍA}

Esta investigación se fundamenta en el paradigma mixto (cuali - cuantitativo), de corte introspectivo vivencial, porque los investigadores participan directamente en la implementación de la metodología PACIE en la Universidad Técnica del Norte en Ecuador. La investigación utiliza el método científico y en el marco de este, es un estudio descriptivo de corte transversal que se desarrolla en tres fases: diseño de la investigación, recolección de información, análisis y desarrollo.

\section{Fase1 - Diseño de la investigación}

En este estudio se determinó como población a los docentes, estudiantes y administrativos del Departamento de Informática de la universidad objeto de estudio, de los cuales la mayoría son mujeres. Se realizó el cálculo de la muestra representativa de la población quedando las cifras como se observa en la Tabla 1.

Tabla 1: Muestra representativa de la población.

\begin{tabular}{|c|c|c|}
\hline \multirow{2}{*}{ Género } & Mujer & 128 \\
\cline { 2 - 3 } & Hombre & 120 \\
\hline \multicolumn{2}{|c|}{ Total General } & 248 \\
\hline
\end{tabular}

Para el efecto de este estudio se utilizaron las técnicas e instrumentos investigativos revisión documental, encuesta, entrevistas y triangulación de la información.

Fase2 - Recolección de la información

La recolección de la información se llevó a cabo en el primer trimestre del 2015. Se realizó la revisión documental en Google Scholar y en las bases de datos indexadas Ebsco, Scopus, SCimago, Scielo y ProQuest a las cuales se accedió mediante el acceso virtual de la biblioteca UTN. Se obtuvo 55 referencias relacionadas con el tema de estudio, con el empleo de fichas de trabajo se seleccionaron las ideas didácticas, pedagógicas y tecnológicas más relevantes a fin de identificar las potencialidades de la aplicación de la metodología PACIE en un entorno virtual de aprendizaje.

Con la finalidad de determinar el interés que tienen los estudiantes y docentes de la comunidad universitaria en actualizar los conocimientos (científicos, técnicos, tecnológicos y humanísticos), bajo la modalidad elearning (educación virtual), se aplicó una encuesta online, validada por tres expertos, ellos evaluaron la claridad, comprensión, pertinencia, relevancia, orden y número de preguntas. El cuestionario se conformó con 11 preguntas de opción múltiple. Para el análisis de los datos se utilizó el paquete estadístico de investigación social SPSS donde a través del cruce de variables se logró establecer los resultados de la investigación.

Posteriormente, se realizaron entrevistas semi estructuradas a los docentes y administrativos del Departamento de Informática, considerados expertos, con el propósito de recopilar información que permitió establecer los requerimientos necesarios para el diseño, desarrollo e implementación de un campus educativo virtual basado en la metodología PACIE que aporta al desarrollo del pensamiento, a la generación y divulgación de conocimientos de forma autónoma, constructivista y colaborativa. Finalmente, se efectuó la triangulación de la información obtenida con la finalidad de eliminar información redundante y conseguir una perspectiva representativa sobre las potencialidades de la metodología PACIE en la Educación Virtual desde la experiencia de la Universidad Técnica del Norte.

\section{Fase3 - Análisis y desarrollo}

Con los datos obtenidos en la fase 1 y 2 se estableció la estructura de la metodología PACIE y sus potencialidades en la modalidad e-learning (aprendizaje virtual), su perspectiva metodológica se fundamenta en el uso adecuado de las tecnologías con un enfoque constructivista para incentivar el trabajo autónomo, crítico, reflexivo, colaborativo y cooperativo. Estos aspectos se circunscriben en la participación e interacción social entre todos los que conforman la comunidad virtual a fin de construir aprendizajes significativos.

\section{RESULTADOS Y DISCUSIÓN}

En base al análisis documental en las 55 referencias relacionadas con el tema de estudio se logró esclarecer y describir dos variables: 1) la estructura de la metodología PACIE y 2) sus potencialidades. En cuanto a la 
estructura de la metodología PACIE, el Campus Educativo Virtual de la UTN contempló cinco fases, las mismas que hacen referencia a las siglas de PACIE: Presencia (P), en esta fase se desarrolló la imagen corporativa del Entorno Virtual de Aprendizaje (EVA) considerando el uso correcto de las TIC para propiciar la navegación e interacción de forma sencilla, intuitiva, amigable para los usuarios. Ver Figura 1.

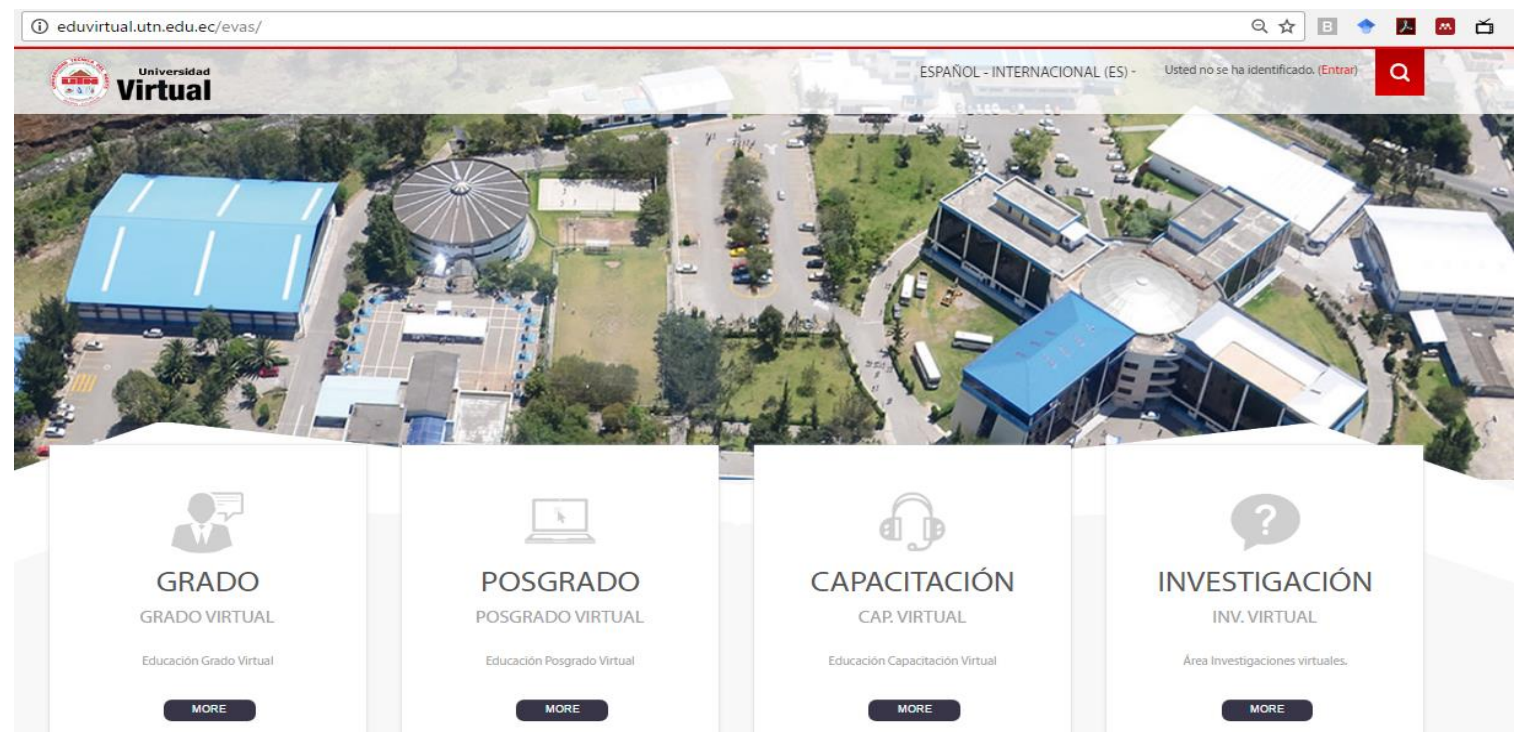

Fig. 1: Presencia del Campus Educativo Virtual UTN.

Alcance (A), en base a los resultados obtenidos en la Fase2, se diseñaron los programas de aprendizaje con estructura secuencial y objetivos claramente definidos bajo un enfoque de pensamiento intuitivo-vivencial con estándares y destrezas pedagógicas. Ver Figura 2.

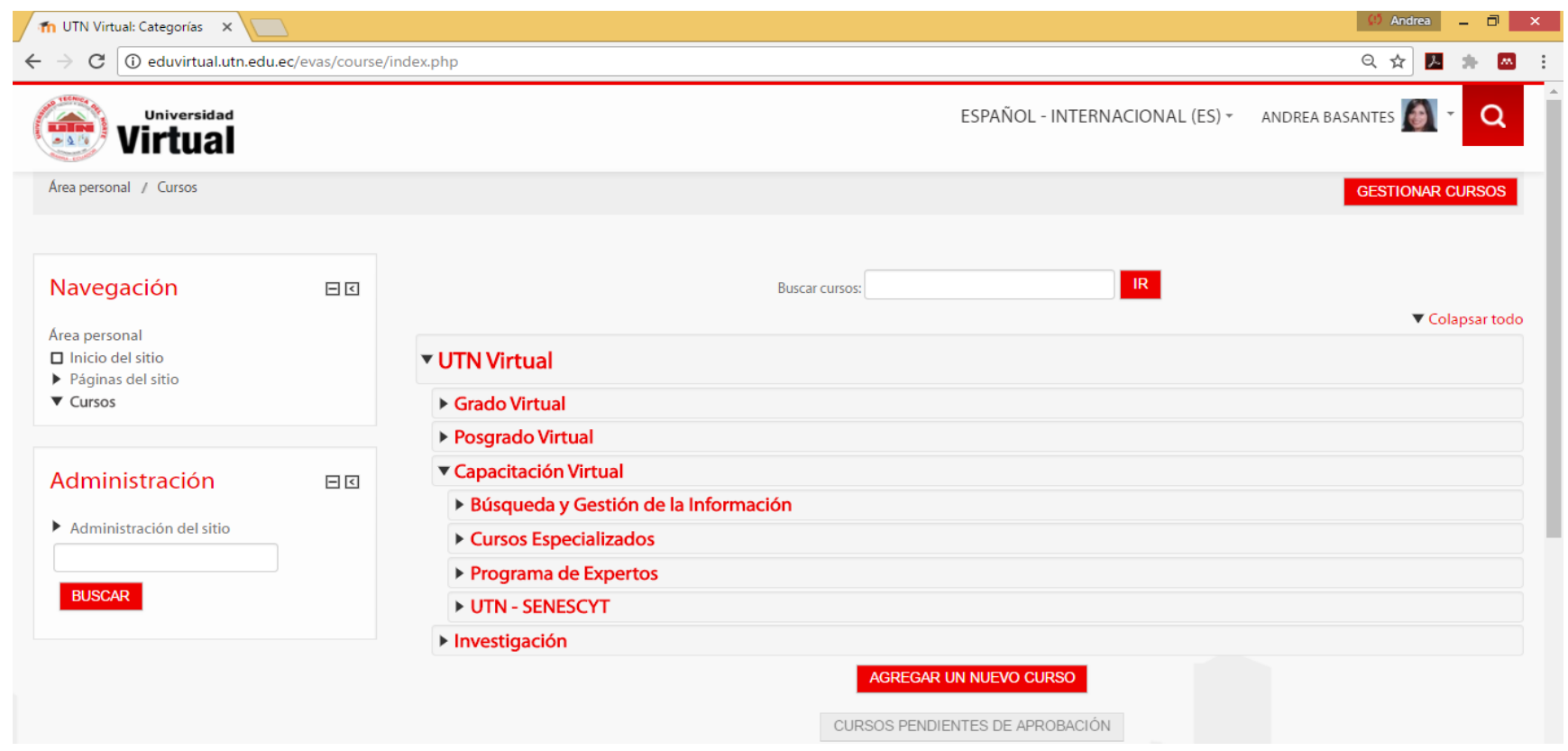

Fig. 2: Programas de aprendizaje online.

Capacitación (C), se realizó la formación de docentes como tutores virtuales con el propósito de dar a conocer las potencialidades de la metodología PACIE, el nuevo rol docente, las funciones que debe cumplir, las estrategias que debe emplear, en sí, las competencias asociadas al nuevo contexto de formación en línea desde la perspectiva docente/tutor. La generación de conocimientos es posible en el marco de interacción humana y las enseñanzas intersubjetivas.

Interacción (I), se seleccionaron los recursos y actividades de la web 2.0 y 3.0 para socializar, compartir y generar la interacción, de tal manera que el estudiante se sienta acompañado y muestre su motivación e interés mediante la participación en línea, fomentando el aprendizaje colaborativo y cooperativo para construir conocimientos. 
E-learning $(\mathrm{E})$, como base del proceso virtual se emplearon las tecnologías existentes en la UTN y en la web para automatizar las actividades y evaluaciones sin descuidar el enfoque pedagógico, constructivista y sociocrítico para lograr conocimientos en situaciones universalistas.

Una vez creado el Campus Educativo Virtual UTN, se realizó la implementación de la metodología PACIE en las aulas virtuales para cada uno de los programas desarrollados en la Fase Alcance. La estructura de la metodología PACIE se centró en la creación de tres bloques:

Bloque 0 o PACIE, es el más importante dentro del proceso metodológico y permitió desarrollar la interacción dentro del aula virtual. Este se compone con tres secciones: sección de información, donde se mostró información general del curso, una guía para iniciar el curso, quién es el tutor y la rúbrica de evaluación del curso; por otro lado, la sección de comunicación se destinó para presentar todos los anuncios, noticias y novedades del desarrollo del curso (se recomendó revisar este espacio frecuentemente). Finalmente, la sección de interacción fue creada para resolver inquietudes e inconvenientes de forma colaborativa y cooperativa con toda la comunidad virtual del curso.

Bloque Académico, es este se desarrollaron los contenidos del curso, los cuales son presentados en diferentes formatos para diversificar la exposición temática. Se estructuró en cuatro secciones: sección de exposición, la cual contiene la información que necesita conocer el estudiante, cuidando que no sea repetitiva para no cansar, aburrir y desertar al estudiante; la sección de rebote, constituye un filtro con actividades para compartir conocimientos de forma crítica y reflexiva a través de foros, chat, videoconferencias, blogs entre otros recursos de la web o plataforma Moodle, pues de esta manera el estudiante debe leer y asimilar la información de la sección de exposición, en caso contrario no podrá continuar con las actividades planificadas. En la tercera sección de construcción el tutor no interactúa, permite que los estudiantes investiguen, exploren, resuelvan, aprendan haciendo y si se equivocan deben corregir y superar las actividades propuestas. La última sección de comprobación tiene por objetivo evaluar el aprendizaje del estudiante a fin de conocer si aprendió, asimiló y comprendió los contenidos propuestos.

Bloque de Cierre, se caracteriza porque permite culminar las tareas pendientes o cerrar procesos inconclusos del curso o de la labor tutorial. Se construyeron tres secciones: sección de negociación, en este espacio se propició la comunicación con el tutor para entregar trabajos atrasados o trabajos que no cumplieron la comprobación de destrezas, además se incorporó un foro de despedida en el aula virtual en el que pueden interactuar todos los participantes. Finalmente, la sección de retroalimentación, el cual es un espacio destinado para conocer la opinión de los participantes en cuanto al desarrollo de los contenidos, la labor tutorial, el uso de la tecnología, entre otros aspectos que sirvieron para mejorar la calidad educativa en la modalidad virtual. A continuación, en la Figura 3, se muestra en forma resumida los tres bloques creados para cada uno de los programas académico en el aula virtual de Moodle:

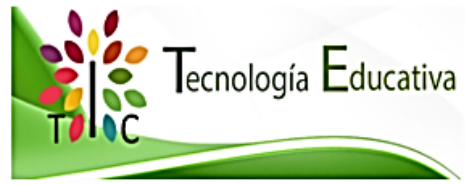

$$
\begin{aligned}
& \text { Area de información } \\
& \text { E Guía: Conociendo el aula } \\
& \text { 国 ¿Quién es la tutora? } \\
& \text { Eúbrica de evaluación } \\
& \text { 郭 Area de comunicación } \\
& \text { [๑. Mi Cartelera } \\
& \text { Area de interacción } \\
& \text { ¿ Apoyo "SOS" } \\
& \text { Cᄂ. Café virtual } \\
& \text { Novedades }
\end{aligned}
$$
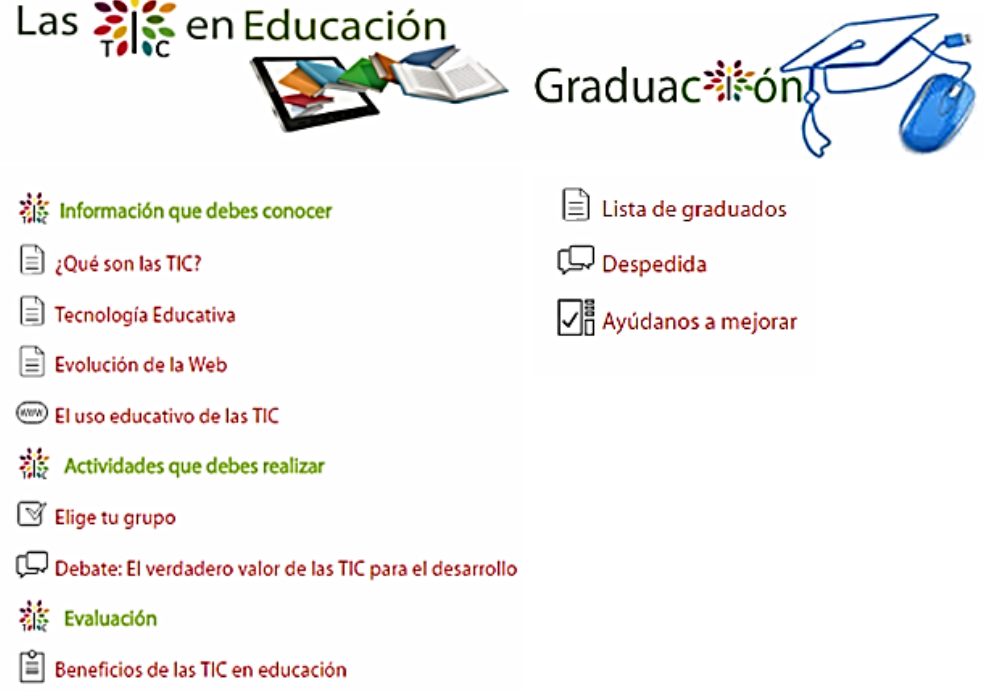

Fig. 3: Bloques metodología PACIE.

Así mismo, la revisión bibliográfica permitió identificar las potencialidades de la metodología PACIE en los entornos virtuales de aprendizaje, estas hacen referencia al fortalecimiento del programa curricular online, al trabajo colaborativo y la humanización a través de la calidad y calidez humana. Estas potencialidades, se 
contrastaron con los resultados de las entrevistas realizadas a los docentes y personal administrativo del Departamento de Informática de la UTN sobre la experiencia de la aplicación de la metodología PACIE en el Campus Educativo Virtual. Los entrevistados mostraron su satisfacción al contar con programas curriculares online bien definidos, con suficiente tecnología que promueve el trabajo colaborativo y con la calidad y calidez humana reflejada por los tutores virtuales. Por otra parte, manifestaron que esta metodología les permite estar actualizados y a la altura de la era digital, de tal forma que, facilita el proceso formativo mediante E-learning. En general consideran pertinente e importante la implementación de la metodología PACIE en el entorno virtual de aprendizaje favoreciendo la organización académica, pedagógica, tecnológica y comunicacional. Se colige que las potencialidades de la metodología PACIE permiten apoyar, comunicar, interactuar y educar en la virtualidad.

Por otra parte, en la descripción general de la muestra de 248 estudiantes del entorno virtual, se encontró que el $51,61 \%$ son mujeres y $48,39 \%$ hombres, mientras que el rango de edad mínima es de 21 años y la máxima de 41 años, con una media de 31 a 35 años. Los resultados obtenidos en la encuesta aplicada a los estudiantes del entorno virtual arrojaron los factores decisivos que inciden en ellos para matricularse en los cursos online. Entre estos factores relevantes se destacan la metodología educativa, la capacitación docente, el prestigio institucional, los documentos de apoyo y el tiempo de duración. La metodología PACIE a través de cada una de sus fases permite potenciar dichos factores; es decir, la metodología educativa, tiempo de duración y el prestigio Institucional se definen en la Fase Alcance, la capacitación docente mediante la Fase de Capacitación y el último factor, los documentos de apoyo en la Fase se Interacción, lo cual fortalece la propuesta educativa virtual.

Para determinar el nivel de significancia, las preguntas fueron construidas bajo la escala Likert, se estableció cinco categorías con su código y valor respectivo; a fin de que las categorías se vuelvan mutuamente excluyentes se determinaron rangos tal como se detalla en la Tabla 2. Después del procesamiento de datos, todos los criterios (prestigio institucional, capacitación docente, metodología educativa, documentos de apoyo y tiempo de duración) tienen un valor ponderado (Vp) que oscila entre 4,36 y 4,42. Ver Tabla 3.

Tabla 2: Escala likert.

\begin{tabular}{|l|c|c|c|c|}
\hline \multicolumn{1}{|c|}{ Categoría } & Código & Valor & \multicolumn{2}{c|}{ Rangos } \\
\hline Muy Importante & $\mathrm{Ml}$ & 5 & 4,51 & 5 \\
\hline Importante & $\mathrm{I}$ & 4 & 3,51 & 4,50 \\
\hline Medianamente Importante & Med. I & 3 & 2,51 & 3,50 \\
\hline Casi Nada Importante & $\mathrm{CNI}$ & 2 & 1,51 & 2,50 \\
\hline Nada Importante & $\mathrm{NI}$ & 1 & 0 & 1,50 \\
\hline
\end{tabular}

Tabla 3: Criterios que inciden para matricularse en los cursos online.

\begin{tabular}{|c|c|c|c|c|c|c|c|c|}
\hline \multicolumn{1}{|c|}{ Criterios } & $M I$ & $I$ & Med. I & CNI & NI & \multirow{2}{*}{ Total } & \multirow{2}{*}{ Vp } & \multirow{2}{*}{$C$} \\
\cline { 2 - 9 } Significancia & 5 & 4 & 3 & 2 & 1 & & \\
\hline Prestigio Institucional & 165 & 47 & 17 & 7 & 12 & 248 & 4,4 & I \\
\hline Capacitación Docentes & 159 & 55 & 18 & 8 & 8 & 248 & 4,41 & I \\
\hline Metodología Educativa & 162 & 58 & 12 & 3 & 13 & 248 & 4,42 & I \\
\hline Documentos de Poyo & 148 & 65 & 19 & 8 & 8 & 248 & 4,36 & I \\
\hline Tiempo de Duración & 124 & 74 & 32 & 4 & 14 & 248 & 4,17 & I \\
\hline
\end{tabular}

El valor ponderado permitió priorizar el orden de los criterios y el nivel de significancia en el género para matricularse en un curso virtual. Ver Tabla 4. Coincidiendo con los resultados de la UNESCO (2010) y Prieto (2016) se requiere de una adecuada metodología educativa para dinamizar el aprendizaje y desarrollar las capacidades humanas en los más variados contextos; la metodología PACIE. En cuanto a las potencialidades que promueve la metodología PACIE en el paradigma de educación virtual fue necesario establecer una escala de medida compuesta por cinco categorías, estas permitieron conocer la relación que sienten los estudiantes con el aspecto del fortalecimiento del programa curricular online, el trabajo colaborativo y la calidad y calidez humana en el proceso curricular online. Luego del procesamiento de datos, todas las variables (potencialidades de PACIE) tienen un valor ponderado (Vp) que oscila entre 4,74 y 4,78 . (Tabla 5)

En consideración a estos resultados, los estudiantes del entorno virtual expresaron que todas las potencialidades que promueve PACIE son muy importantes. En orden de importancia los estudiantes destacaron la calidad y calidez humana, el programa curricular online y por último el trabajo colaborativo. 
Finalmente, cabe señalar que este estudio reafirma las potencialidades de la metodología PACIE y concuerda con los resultados del estudio realizado por Ferrer y Bravo (2017) en el que manifiesta que, la metodología PACIE aporta beneficios en el ámbito organizacional, académico, pedagógico, comunicacional y tecnológico. Cabe señalar que al momento no se reportan estudios sobre inconvenientes o limitantes para la aplicación de la metodología PACIE en los Entornos Virtuales de Aprendizaje; al contrario, se destacan las potencialidades en la educación virtual.

Tabla 4: Priorización de criterios y significancia en el género.

\begin{tabular}{|l|c|c|c|}
\hline \multirow{2}{*}{ Categoría } & \multirow{2}{*}{ Vp } & \multicolumn{2}{c|}{ Género } \\
\cline { 3 - 4 } & & Mujeres & Hombres \\
\hline Metodología & 4,42 & 2,28 & 2,14 \\
\hline Docentes & 4,41 & 2,28 & 2,13 \\
\hline Prestigio & 4,40 & 2,27 & 2,13 \\
\hline Documentos de Poyo & 4,36 & 2,25 & 2,11 \\
\hline Tiempo de Duración & 4,17 & 2,15 & 2,02 \\
\hline
\end{tabular}

Tabla 5: Ponderación potencialidades de PACIE

\begin{tabular}{|c|c|c|c|c|c|c|c|c|}
\hline \multirow{2}{*}{$\underbrace{\text { Variables }}_{\text {Significancia }}$} & MI & 1 & $\begin{array}{c}\text { Med. } \\
\quad\end{array}$ & $\mathrm{CNI}$ & $\mathrm{NI}$ & \multirow{2}{*}{ Total } & \multirow{2}{*}{$V p$} & \multirow{2}{*}{ Categoría } \\
\hline & 5 & 4 & 3 & 2 & 1 & & & \\
\hline Programa curricular online & 212 & 19 & 10 & 7 & 0 & 248 & 4,76 & $\mathrm{Ml}$ \\
\hline Trabajo colaborativo & 198 & 40 & 5 & 5 & 0 & 248 & 4,74 & $\mathrm{Ml}$ \\
\hline Calidad y Calidez humana & 201 & 39 & 8 & 0 & 0 & 248 & 4,78 & MI \\
\hline \multicolumn{7}{|c|}{ Promedio Vp } & 4,76 & $\mathrm{Ml}$ \\
\hline
\end{tabular}

\section{CONCLUSIONES}

Las evidencias derivadas de este estudio descriptivo permiten enumerar las siguientes conclusiones que afirman las potencialidades de la metodología PACIE en el entorno virtual de la enseñanza universitaria en la Universidad Técnica del Norte del Ecuador: 1) La aplicación correcta de las cinco fases de la metodología PACIE favorece la organización académica, pedagógica, tecnológica y comunicacional del proceso de enseñanza-aprendizaje. 2) Las personas que optan por los cursos online consideran como prioridad la metodología educativa, la capacitación docente, el prestigio institucional, los documentos de apoyo, el tiempo de duración, siendo estos aspectos favorecidos por la metodología PACIE. 3) La aplicación de la metodología PACIE en el paradigma de educación virtual potencializa el programa curricular online, el trabajo colaborativo y promueve la calidad y calidez humana mediante la interacción entre los estudiantes.

\section{REFERENCIAS}

Acosta, L., Abreu, O. y Coronel, M. Sistema de Formación Pedagógica en la Universidad de Otavalo en Ecuador. doi: 10.4067/S0718-50062015000200007. Formación Universitaria, 8(2), 43-52 (2015)

Arocena, R. y Sutz, J. Foro Cilac 2016, Eje Temático: Universidades para el desarrollo. En línea: https://goo.gl/K6EUcv, acceso: 20 noviembre 2016 (2016)

Basantes, A., Naranjo, M., Gallegos, M. y Benítez, N. Los Dispositivos Móviles en el Proceso de Aprendizaje de la Facultad de Educación Ciencia y Tecnología de la Universidad Técnica del Norte de Ecuador, doi: 10.4067/S071850062017000200009. Formación Universitaria, 10(2), 79-88 (2017)

Cano, O. Antecedentes internacionales y nacionales de las TIC a nivel superior: su trayectoria en Panamá. Revista Electrónica" Actualidades Investigativas en Educación", 12(3), (2012)

Castro, P. y González-Palta, I. Percepción de Estudiantes de Psicología sobre el Uso de Facebook para Desarrollar Pensamiento Crítico, doi: 10.4067/S0718-50062016000100006. Formación Universitaria, (en línea), 9(1), 45-56 (2016)

Cózar-Gutiérrez, R., De Moya-Martínez, M., Hernández-Bravo, J., y Hernández-Bravo, J. Conocimiento y Uso de las Tecnologías de la Información y las Comunicaciones (TIC) según el Estilo de Aprendizaje de los Futuros Maestros, doi:10.4067/S0718-50062016000600010. Formación Universitaria, (en línea), 9(6), 105-118 (2016)

Ferrer, K. y Bravo, M. Metodología Pacie en los ambientes virtuales de aprendizaje para el logro de un aprendizaje colabrativo. Revista Electrónica Diálogos Educativos, 12(24), 3-17 (2017) 
Flores, D. Alternativas didácticas y el aprendizaje autónomo en la asignatura computación aplicada a la educación en la universidad Tecnológica Equinoccial, extensión Santo Domingo. Tesis de Magister, Dpto. Ciencias Informáticas, Universidad Regional Autónoma de los Andes, Ambato, Ecuador (2017)

García, M. El uso de e-learning: un enfoque general. Encuentro, (66), 78-83 (2017)

Jordan, K. MOOC Completion Rates: The Data. En línea: https://goo.gl/iZdbTt, acceso: 17 noviembre 2016. (2015)

Lerner, J. y Gil, L. Metodología del aprendizaje: una experiencia analítica en el aula, 1aㅡ Ed., 49-65, Universidad Eafit, Medellín, Colombia (2006)

Marsiske, R. La universidad latinoamericana en el siglo XX: una aproximación. Universidades, 66(65), (2015)

Martínez, C. y Fernández, M. El uso de Moodle como entorno virtual de apoyo a la enseñanza presencial. La práctica educativa en la Sociedad de la Información: Innovación a través de la investigación, 291-300 (2011)

Martínez, E. y Fuentes, M. Implementación de la metodología PACIE en cursos semipresenciales en el Entorno Virtual de Aprendizaje de TIC. Revista Iberoamericana de Producción Académica y Gestión Educativa, ISSN: 2007-8412, 2(2), 1$12(2014)$

Mejía, J. y López, D. Modelo de Calidad de E-learning para Instituciones de Educación Superior en Colombia, doi: 10.4067/S0718-50062016000200007. Formación Universitaria, (en línea), 9(2), 59-72 (2016)

Micheli, J. y Armendáriz, S. Estructuras de Educación Virtual en la Organización Universitaria: Un Acercamiento a la Sociedad del Conocimiento, doi: 10.4067/S0718-50062011000600005. Formación Universitaria, (en línea), 4(6), 35-48 (2011)

Núñez, E. Diseño de un Complejo Educativo Virtual. Actas del 40 Congreso Virtual Educa, 130-142, Lima-Perú, 20 a 22 de abril (2015)

Oñate, Luis. La Metodología PACIE. FATLA (Fundación para la Actualización Tecnológica de Latinoamérica). En línea: http://bit.ly/2vQPXEA, acceso: 10 septiembre 2016 (2009)

OCDE / CEPAL / CAF. Perspectivas económicas de América Latina 2017. Juventud, competencias y emprendimiento. OECD Publishing, Paris. http://dx.doi.org/10.1787/leo-2017-es. En línea: https://goo.gl/rCr6ch. Acceso: 3 noviembre 2016, París (2016)

OREALC. /2014 /PI /H /1. América Latina y el Caribe: Revisión Regional 2015 de la Educación para Todos. En línea: https://goo.gl/8YGmg5. Acceso: 20 septiembre 2017 (2014)

Prieto, J. Una aproximación metodológica al uso de redes sociales en ambientes virtuales de aprendizaje para el fortalecimiento de las competencias transversales de la Universidad EAN. Virtu@Imente, 1(1), 1-16 (2016)

Rocha, R. Escala de opinión de los estudiantes sobre la efectividad de la docencia (EOEED) en Educación Superior, doi: 10.4067/S0718-50062013000600003. Formación Universitaria, (en línea), 6 (6), 13-22 (2013)

Sánchez, G. Paradigma de la educación virtual y los nuevos escenarios de aprendizaje. Educación Superior, (21), (2017)

UNESCO. La UNESCO y la educación superior, 2014-2017: aportes de la Reunión de Cátedras UNESCO sobre la educación superior, las TIC en la educación y los profesores (Sylvie Didou Aupetit). En línea: https://goo.gl/oNqgLK. Acceso: 20 septiembre 2016. (2014)

UNESCO. Logro Cognitivo de los estudiantes en América Latina y el Caribe. Salesianos Impresores S.A., Chile (2010)

Yamba-Yugsi, M. y Luján-Mora, S. Cursos MOOC: Factores que disminuyen el abandono en los participantes. Enfoque UTE, 8(1), 1-15 (2017) 
